\title{
Single-term Walsh series method for solving Volterra's population model
}

\author{
Behnam Sepehrian \\ Department of Mathematics, Faculty of Science, Arak University, Arak 38156-8-8349, Iran \\ Email: b-sepehrian@araku.ac.ir
}

Copyright (C)2014 Behnam Sepehrian. This is an open access article distributed under the Creative Commons Attribution License, which permits unrestricted use, distribution, and reproduction in any medium, provided the original work is properly cited.

\begin{abstract}
Single-term Walsh series are developed to approximate the solution of the Volterra's population model. Volterra's model is a nonlinear integro-differential equation where the integral term represents the effect of toxin. Properties of Single-term Walsh series are presented and are utilized to reduce the computation of the Volterra's population model to some algebraic equations. The method is computationally attractive, and applications are demonstrated through illustrative examples. A comparison is made with existing results.
\end{abstract}

Keywords: Numerical methods, STWS method, Volterra's population model.

\section{Introduction}

In the present paper we are concerned with the numerical solution of the Volterra model for population growth of a species within a closed system. This equation is given in $[9,15]$ as

$\kappa u^{\prime}(t)=u-u^{2}-u \int_{0}^{t} u(x) d x, \quad u(0)=u_{0}$,

where $u(t)$ is the scaled population of identical individuals at time $t$, and $\kappa=\frac{c}{a b}$ is a prescribed nondimensional parameter. Moreover, $a>0, b>0$ and $c>0$ are the coefficients of the birth rate, crowding and toxicity, respectively. The analytical solution [13]

$u(t)=u_{0} \exp \left(\frac{1}{\kappa} \int_{0}^{t}\left[1-u(\tau)-\int_{0}^{\tau} u(x) d x\right] d \tau\right)$,

shows that $u(t)>0$ if $u_{0}>0$ for all $t$. The mathematical behavior of the rapid growth, $u(t)$, was introduced by Scudo [9] and justified by Small[13]. It was shown in [9] and [13] that for the case $\kappa<<1$, a rapid rise occurs along the logistic curve that will reach a peak and be followed by a slow exponential decay where $u(t) \rightarrow 0$ as $t \rightarrow \infty$. And, for $\kappa$ large, $u(t)$ has a smaller amplitude compared to the amplitude of $u(t)$ for the case $\kappa<<1$.

Several approximate and numerical solutions for Volterra's population model are known. Wazwaz [15] implemented the series solution method and decomposition method independently to Eq. (1) and to a related ordinary differential equation. Furthermore, the pade approximations was used in the analysis to capture the essential behavior of the population $u(t)$ of identical individuals in [15]. In [5], operational matrices of derivative and product 
of Rational Chebyshev functions are introduced and implemented for solving Eq. (1) by using tau method. In [14], three numerical algorithms namely the Euler method, the modified Euler method and the forth order Runge-Kutta method are given and pointwise approximations for the solution of Eq. (1) are obtained.

Walsh functions (WF) have found wide applications in signal processing, communication, and pattern recognition [7]. Rao et al. [6] presented a method of extending computation beyond the limit of the initial normal interval in Walsh series analysis of dynamical systems. In the last method, various time functions in the system were first expanded as truncated WF with unknown coefficients. Then, by using the Kronecker product [4], the unknown coefficient of the rate variable was obtained by finding the inverse of a square matrix. It was shown that this method involves some numerical troubles if the dimension of this matrix is large. Hsiao and Chen [3] introduced the Walsh series operational matrix of integration to solve linear integral equations. Due to the nature of the Walsh functions, the solutions obtained were piecewise constant. Rao et al. [6] introduced single-term Walsh series (STWS) to remove the inconveniences in WF technique. Furthermore, Balachandran and Murugesan [1,2] applied STWS technique to the analysis of linear and nonlinear singular systems. Sepehrian and Razzaghi [10,11] implemented STWS method to the bilinear and nonlinear time varying singular systems. Also, STWS technique was applied to solve the nonlinear Volterra-Hammerstein integral equations in [12].

In the present article, we introduce a new computational method for solving Eq. (1). The method presented here is computationally very attractive. It does not require the Kronecker product of matrices and there is no need for any operational matrices. One main merit of using this technique is that it provides both pointwise and block-Pulse approximations for solution of (1).

The paper is organized as follows. Section 2 is devoted to the basic formulation of WF and STWS required for our subsequent development. In Section 3, the solution of (1) using STWS is considered. Section 4 is devoted to numerical examples. In numerical examples we demonstrate the accuracy of the proposed scheme by comparing the our numerical findings with the existing results and the exact solutions.

\section{Properties of WF and STWS}

\subsection{Walsh functions}

A function $\mathrm{f}(\mathrm{t})$, integrable in $[0,1)$, may be approximated using $\mathrm{WF}$ as

$f(t)=\sum_{i=0}^{\infty} f_{i} \phi_{i}(t)$,

where $\phi_{i}(t)$ is the $i$ th WF and $f_{i}$ is the corresponding coefficient. In practice, only the first $m$-term WF are considered, where $m$ is an integral power of 2. Then from Eq. (2) we get

$f(t)=\sum_{i=0}^{m-1} f_{i} \phi_{i}(t)=F^{T} \Phi(t)$,

where

$F=\left(f_{0}, f_{1}, \cdots, f_{m-1}\right)^{T}$

and

$\Phi(t)=\left(\phi_{0}(t), \phi_{1}(t), \cdots, \phi_{m-1}(t)\right)^{T}$.

The coefficients $f_{i}$ are chosen to minimize the mean integral square error

$\epsilon=\int_{0}^{1}\left(f(t)-F^{T} \Phi(t)\right)^{2} d t$,

and are given by

$f_{i}=\int_{0}^{1} f(t) \phi_{i}(t) d t$.

The integration of the vector $\Phi(t)$ defined in Eq. (3) can be approximated by

$\int_{0}^{t} \Phi\left(t^{\prime}\right) d t^{\prime} \simeq E \Phi(t)$,

where $E_{m \times m}$ is the $m \times m$ operational matrix for integration with $E_{1 \times 1}=\frac{1}{2}$ and is given in [8]. 


\subsection{Single-term Walsh series}

With the STWS approach, in the first interval, the given function is expanded as single- term Walsh series in the normalized interval $\tau \in[0,1)$, which corresponds to $t \in\left[0, \frac{1}{m}\right)$ by defining $\tau=m t, m$ being any integer. In STWS, the matrix $E$ in Eq. (4) becomes $E=\frac{1}{2}$.

Let $u(\tau)$ and $u^{\prime}(\tau)$ expanded by STWS series in the first interval as

$u(\tau)=U^{(1)} \phi_{0}(\tau)$,

and

$u^{\prime}(\tau)=V^{(1)} \phi_{0}(\tau)$.

By using Eq. (5) and integrating Eq. (6) with $E=\frac{1}{2}$ we get

$U^{(1)}=\frac{1}{2} V^{(1)}+u(0)$,

and therefore

$V^{(1)}=2\left(U^{(1)}-u(0)\right)$,

where $u(0)$ is the initial condition. Also, according to Sannuti [8] we have

$u(1)=\int_{0}^{1} u^{\prime}(\tau) d \tau+y(0)=V^{(1)}+y(0)$.

By (6) and (8), we get

$u(1)=2 U^{(1)}-u(0)$.

\section{Solution of Volterra's population model}

In order to use STWS, we first divide interval $[0,1)$ to $m$ equal subintervals, where $m$ is any positive integer. We then transform each interval $\frac{j-1}{m} \leq x \leq t<\frac{j}{m}, j=1, \cdots, m$, into $[0,1)$ by means of transformations $\tau_{j}=m t-(j-1)$ and $\lambda_{j}=m x-(j-1)$. Using Eq. (1), in the first interval we have

$\kappa u^{\prime}\left(\tau_{1}\right)=\frac{1}{m} u-\frac{1}{m} u^{2}-\frac{1}{m^{2}} u \int_{0}^{\tau_{1}} u\left(\lambda_{1}\right) d \lambda_{1}, u(0)=u_{0}$,

and similarly in $i$ th interval we have

$\kappa u^{\prime}\left(\tau_{i}\right)=\frac{1}{m} u-\frac{1}{m} u^{2}-\frac{1}{m^{2}} u\left(\sum_{j=1}^{i-1} \int_{0}^{1} u\left(\lambda_{j}\right) d \lambda_{j}+\int_{0}^{\tau_{i}} u\left(\lambda_{i}\right) d \lambda_{i}\right)$.

Let $u\left(\tau_{i}\right)$ and $u^{\prime}\left(\tau_{i}\right)$ are expressed by STWS as

$u\left(\tau_{i}\right)=U^{(i)} \phi_{0}\left(\tau_{i}\right), \quad i=1, \cdots, m$

and

$u^{\prime}\left(\tau_{i}\right)=V^{(i)} \phi_{0}\left(\tau_{i}\right), \quad i=1, \cdots, m$.

Similarly to Eqs. (7) and (9) we get

$V^{(i)}=2\left(U^{(i)}-u(i-1)\right)$,

and

$u(i)=2 U^{(i)}-u(i-1)$.

In above equations $U^{(i)}$ and $u(i)$ give the block-pulse and the discrete values of $u(t)$ respectively. 
Using Eq. (4) with $E=\frac{1}{2}$ and Eqs. (10), (12), (13) and (14), the block-pulse value $U^{(1)}$ in the first interval is given by

$\left(\frac{1}{m}+\frac{1}{2 m^{2}}\right)\left(U^{(1)}\right)^{2}+\left(2 \kappa-\frac{1}{m}\right) U^{(1)}-2 \kappa u(0)=0$.

Similarly by using Eq. (11) in the $i$ th interval we have

$\left(\frac{1}{m}+\frac{1}{2 m^{2}}\right)\left(U^{(i)}\right)^{2}+\left(2 \kappa-\frac{1}{m}+\frac{1}{m^{2}} \sum_{j=1}^{i-1} U^{(j)}\right) U^{(i)}-2 \kappa u(i-1)=0, \quad i=2, \cdots, m$.

By solving Eq. (16) for $U^{(1)}$ and Eq. (17) for $U^{(i)}, i=2, \cdots, m$, the block-pulse and by using Eq. (14) discrete approximations of $u(t)$ can be obtained.

\section{Illustrative example}

We applied the method presented in this paper and solved Eq. (1) for $u(0)=0.1$ and $\kappa=0.02,0.04,0.1,0.2$ and 0.5 and then evaluated $u_{\max }$ and $t_{\text {critical }}$, which also are evaluated in $[5,15]$. The resulting values using the methods in [5] and [15] together with the exact values obtained by using [14]

$u_{\max }=1+\kappa \ln \left(\frac{\kappa}{1+\kappa-u_{0}}\right)$,

are presented in Table 1. In Table 2, our computational results for approximations of $u_{\max }$ with $m=20,100$, and 200 are shown. So, a comparison between methods in [5] and [15] and the present method is made. Fig. 1 and Fig. 2, show us the mathematical behavior of the approximate solutions obtained by STWS method with $m=100$. These graphs agree with the previous studies in [9] and [13].

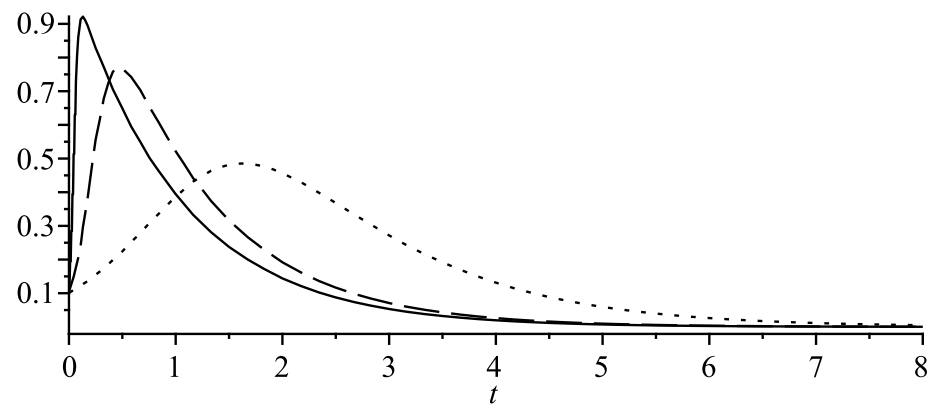

Figure 1: Block-pulse approximations for the cases $\kappa=0.02$ (Solid), 0.1 (Dashes) and 0.5 (Dots).

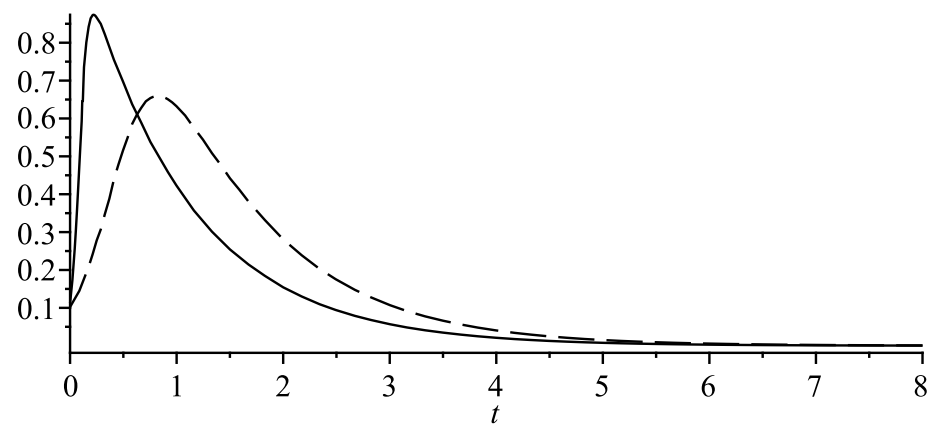

Figure 2: Block-pulse approximations for the cases $\kappa=0.04$ (Solid) and 0.2 (Dashes). 
Table 1: Computational results of methods in [15] and [5] together with the exact values for $u_{\max }$.

\begin{tabular}{ccccc}
\hline$\kappa$ & Critical $t$ & Method in [15] & Method in [5] & Exact $u_{\max }$ \\
\hline 0.02 & 0.111845 & 0.903838 & 0.923463 & 0.923427 \\
0.04 & 0.210246 & 0.861240 & 0.873708 & 0.873720 \\
0.1 & 0.464476 & 0.765113 & 0.769734 & 0.769741 \\
0.2 & 0.816858 & 0.657912 & 0.659045 & 0.659050 \\
0.5 & 1.626711 & 0.485282 & 0.485188 & 0.485190 \\
\hline
\end{tabular}

Table 2: Estimated values of $u_{\max }$ using STWS with $m=20,100$ and 200.

\begin{tabular}{ccccc}
\hline$\kappa$ & Critical $t$ & $\mathrm{~m}=20$ & $\mathrm{~m}=100$ & $\mathrm{~m}=200$ \\
\hline 0.02 & 0.111845 & 0.937866 & 0.923846 & 0.923451 \\
0.04 & 0.210246 & 0.876359 & 0.873794 & 0.873740 \\
0.1 & 0.464476 & 0.769689 & 0.769741 & 0.769741 \\
0.2 & 0.816858 & 0.658853 & 0.659030 & 0.659048 \\
0.5 & 1.626711 & 0.485106 & 0.485187 & 0.485189 \\
\hline
\end{tabular}

\section{Conclusion}

The properties of STWS are used to solve the Volterra's population model. The method provides both pointwise and block-pulse approximations for unknown function. Furthermore, unlike the Walsh series approach, there is no restriction on $m$ and the method does not require the Kronecker product or the inversion of large matrices. Illustrative examples are included to demonstrate the validity and applicability of the technique.

\section{References}

[1] K. Balachandran, K. Murugesan, "Analysis of nonlinear singular systems via STWS method", Int. J. Comput. Math. , Vol.36, No.(1-2), (1990), pp. 9-12.

[2] K. Balachandran, K. Murugesan, "Numerical solution of a singular non-linear system from fluid dynamics", Int. J. Comput. Math., Vol.38, No.(3-4), (1991), pp. 211-218.

[3] C. H. Hsiao, C. F. Chen, "Solving integral equations via Walsh functions", Comput. Electr. Engrg., Vol.6, (1979), pp.279-292.

[4] P. Lancaster, Theory of matrices, Academic Press, New York, 1969.

[5] K. Parand, M. Razzaghi, "Rational Chebyshev tau method for solving Volterra's population model", Appl. Math. Comput., Vol.149, (2004), pp.893-900.

[6] G. P. Rao, K. R. Palanisamy, T. Srinivasan, "Extension of computation beyond the limit of initial normal interval in Walsh series analysis of dynamical systems", IEEE Trans. Automat. Control, Vol.25, No.2, (1980), pp.317-319.

[7] M. Razzaghi, J. Nazarzadeh, "Walsh functions", Wiley Encyclopedia of Electrical and Electronics Engineering, Vol.23, (1999), pp.429-440.

[8] P. Sannuti, "Analysis and synthesis of dynamic systems via block-pulse functions", Proceedings IEE, Vol.124, No.6, (1977), pp.571-596.

[9] F. M. Scudo, "Volterra and theoritical ecology", Theoret. Popul. Biol., Vol.2, (1971), pp.1-23.

[10] B. Sepehrian, M. Razzaghi, "State analysis of time-varying singular bilinear systems by single-term Walsh series", Int. J. Comput. Math., Vol.80, (2003), pp.413-418.

[11] B. Sepehrian, M. Razzaghi, "Solution of time-varying singular nonlinear systems by single-term Walsh series", Math. Prob. Eng., Vol.3, (2003), pp.129-136. 
[12] B. Sepehrian, M. Razzaghi, "Solution of nonlinear Volterra-Hammerstein integral equations via single-term Walsh series method", Math. Prob. Eng., Vol.5, (2005), pp.547-554.

[13] R. D. Small, "Population growth in a closed system and Mathematical Modeling, in: Classroom Notes in Applied Mathematics", SIAM, Philadelphia, PA, (1989), pp.317-320.

[14] K. G. TeBeest, "Numerical and analytical solutions of Volterra's population model", SIAM Rev., Vol.39, (1997), pp.484-493.

[15] A. M. Wazwaz, "Analytical approximation and pade approximation for Volterra's population model", Appl. Math. Comput., Vol.100, (1999), pp.13-25. 